\title{
INFLUENCE OF INDUCED ANISOTROPY ON MAGNETIC PROPERTIES OF THE Fe73.5Cu1Nb3Si13.5B9 ALLOY IN AMORPHOUS AND NANOCRYSTALLINE STATES
}

\author{
V.V. SHULIKA, A.P. POTAPOV and A.E. PATRAKOV \\ Institute of Metal Physics, UD RAS, GSP-170 Ekaterinburg, Russia
}

(Received in final form 28 September 1997)

\begin{abstract}
The influence of thermomagnetic treatments (TMT) on static hysteresis loops and magnetic losses of Fe73.5Cu1Nb3Si13.5B9 in amorphous and nanocrystalline states was studied. It is established that domain structure destabilization of the nanocrystalline alloy at TMT in a high frequency magnetic field results in maximum decrease of magnetic losses and permeability increase, if a high frequency magnetic field is applied to a sample during the alloy transition from the amorphous to the nanocrystalline state.
\end{abstract}

Keywords: Induced magnetic anisotropy; Amorphous and nanocrystalline materials

\section{INTRODUCTION}

A wide spectrum of magnetic properties of the soft magnetic alloys is obtained by using induced anisotropy which appears after thermomagnetic treatment, TMT. Such influence is especially strong in the soft magnetic alloys where crystallographic anisotropy is very small (Glazer et al., 1994) and in the nanocrystalline alloys where its average is zero (Herzer, 1994). Induced anisotropy, originated after TMT in the direct magnetic field, forms the magnetic texture. In this case the magnetic properties along and transverse to the magnetic field direction at TMT 
are strongly different. Induced magnetic anisotropy may also arise in a ferromagnetic material at thermal treatment without the magnetic field. In this case anisotropy is induced in each domain according to a local distribution of magnetization in a sample. Local induced magnetic anisotropy leads to a domain structure stabilization and deterioration of magnetic properties. A new method of domain structure destabilization has been developed by the authors - it is the TMT in a high frequency magnetic field (Glazer et al., 1992). This method leads to the substantial improvement of the magnetic properties of the amorphous alloy. It is of interest to study an opportunity of application of this method to the nanocrystalline Fe73.5Cu1Nb3Si13.5B9 alloy for further improvement of its soft magnetic properties. It is reasonable to study the changes of the magnetic properties of the alloy $\mathrm{FeCuNbSiB}$ in order to obtain additional information about the nature of induced anisotropy.

\section{EXPERIMENTAL}

Amorphous ribbons of $\mathrm{Fe} 73.5 \mathrm{Cu} 1 \mathrm{Nb} 3 \mathrm{Si13.5B} 9$ were obtained by melt quenching on a rotating disk (ribbon thicknesses are $20-25 \mu \mathrm{m}$, width $10 \mathrm{~mm}$ ). Toroidal samples were wound from this ribbon with an outer diameter $30 \mathrm{~mm}$, inner diameter $25 \mathrm{~mm}$. The samples were annealed at $350^{\circ} \mathrm{C}$ for $1 \mathrm{~h}$ in vacuum for release of internal stresses. For obtaining nanocrystalline structure, samples were annealed at $540^{\circ} \mathrm{C}$ for $1 \mathrm{~h}$. In the first series of experiments the TMT in the amorphous samples was carried out at $400^{\circ} \mathrm{C}$ (above Curie point but lower than the crystallization temperature). In the second series of experiments a magnetic field was applied to the sample during the alloy transition from the amorphous to the nanocrystalline state (heating to $540^{\circ} \mathrm{C}$, keeping for $1 \mathrm{~h}$, cooling in the field at the rate $200^{\circ} \mathrm{Ch}^{-1}$ ). In the third series of experiments the TMT was carried out in the nanocrystalline samples at $540^{\circ} \mathrm{C}$. The effectiveness of TMT of the samples of version 1,2 and 3 were compared. The magnetic properties after TMT in a high frequency $(80 \mathrm{kHz})$, alternating $(50 \mathrm{~Hz})$, and direct magnetic field were compared. Static hysteresis loops, magnetic losses and initial magnetic permeability were measured at the frequency of magnetization reversal of $20 \mathrm{kHz}$ and induction $0.2 \mathrm{~T}$. 


\section{RESULTS AND DISCUSSION}

The results of measurements are presented in Figs. 1-4 and Table I. Static hysteresis loops of samples in the amorphous and nanocrystalline states after TMT are compared in Fig. 1.

The first sample was annealed at $350^{\circ} \mathrm{C}$ (hysteresis loop 1) after this it was exposed to TMT at $400^{\circ} \mathrm{C}$ (hysteresis loop 2). The TMT of the second sample was performed during alloy transition from the amorphous to the nanocrystalline state (annealing at $540^{\circ} \mathrm{C}$ and TMT were combined). It is seen that the effectiveness of TMT is higher for the second sample, it has a lower coercivity after annealing in a magnetic field relative to the first sample (hysteresis loop 3). Static hysteresis loops of the nanocrystalline samples of version 2 and 3 after TMT in direct magnetic field are shown in Fig. 2. The first sample was pre-annealed at the temperature of $540^{\circ} \mathrm{C}$. Annealing at $540^{\circ} \mathrm{C}$ and simultaneous

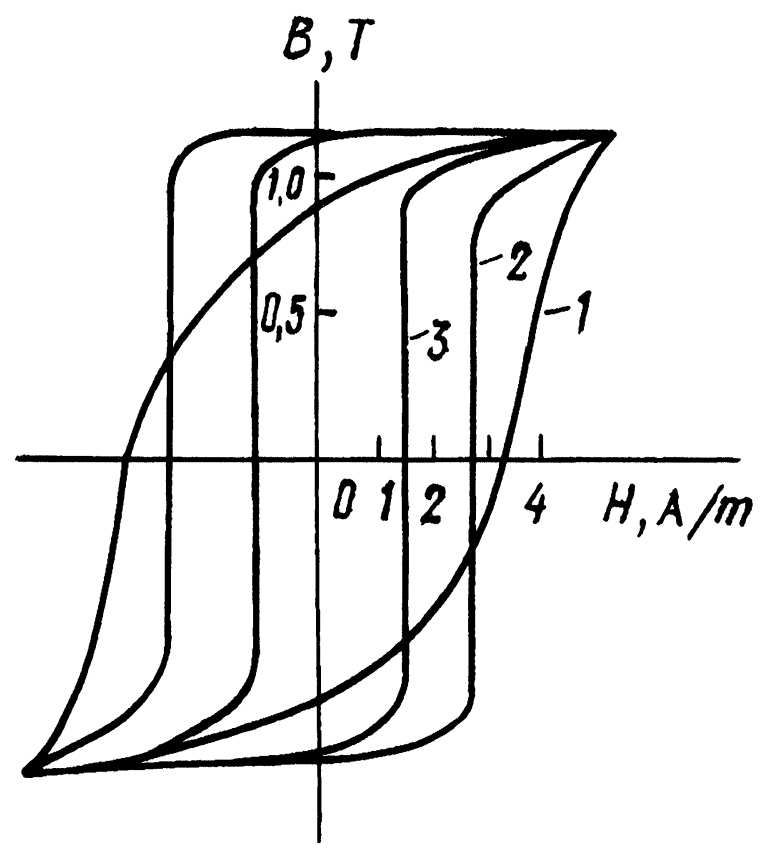

FIGURE 1 The hysteresis loops of samples: after annealing at $350^{\circ} \mathrm{C}(1)$, subsequent TMT in a direct magnetic field at $400^{\circ} \mathrm{C}(2)$, annealing at $540^{\circ} \mathrm{C}$ and simultaneous TMT in a direct magnetic field. 


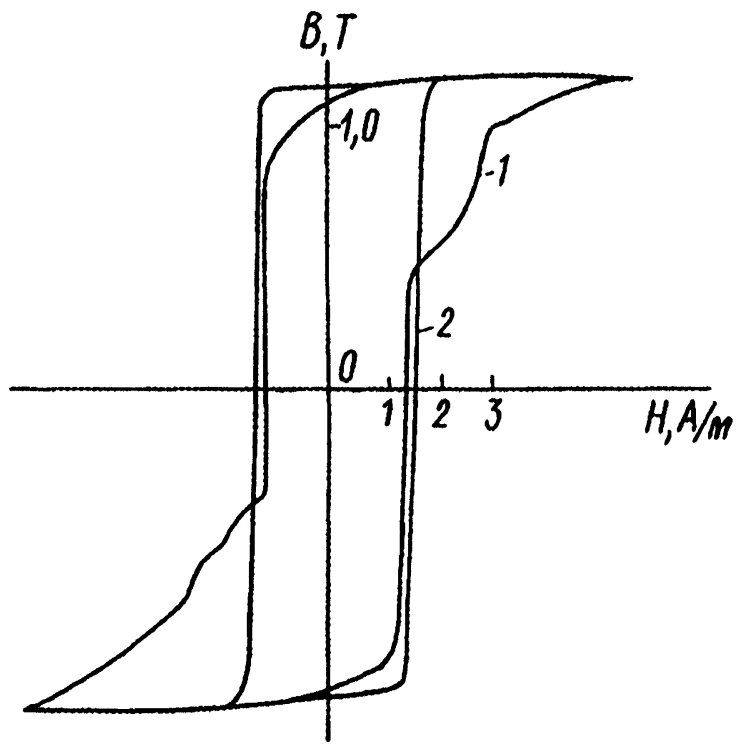

FIGURE 2 The hysteresis loops of samples after annealing at $540^{\circ} \mathrm{C}$ and subsequent TMT in a direct field (1), annealing at the same temperature and simultaneous TMT in a direct field (2).

TMT in direct magnetic field were performed for the second sample. It is seen that the second sample has a more rectangular hysteresis loop in comparison with the first sample, that is TMT is more effective if a magnetic field is applied during a phase transition. Static hysteresis loops of the nanocrystalline samples after annealing in a high frequency $(80 \mathrm{kHz})$ (loop 1), alternating $(50 \mathrm{~Hz})($ loop 2) and direct magnetic field (loop 3) are compared in Fig. 3. Their magnetic properties (initial permeability $\mu_{0}$; maximum permeability $\mu_{\max }$; rectangularity coefficient $\mathrm{Br} / \mathrm{Bm}$, and magnetic losses $P_{0.2 / 20000}$ ) are summarized in Table I.

It is necessary to emphasize that TMT was performed at the temperature of alloy transition from the amorphous to the nanocrystalline state. TMT in a high frequency magnetic field leads to a nonrectangular hysteresis loop, to higher initial permeability, lower coercivity and lower magnetic losses in comparison with TMT in direct or alternating $(50 \mathrm{~Hz})$ magnetic fields.

Figure 4 shows the static hysteresis loops of two samples: (1) after annealing at $540^{\circ} \mathrm{C}$ and subsequent quenching in water from the Curie point; (2) after TMT in a high frequency magnetic field. It follows from 


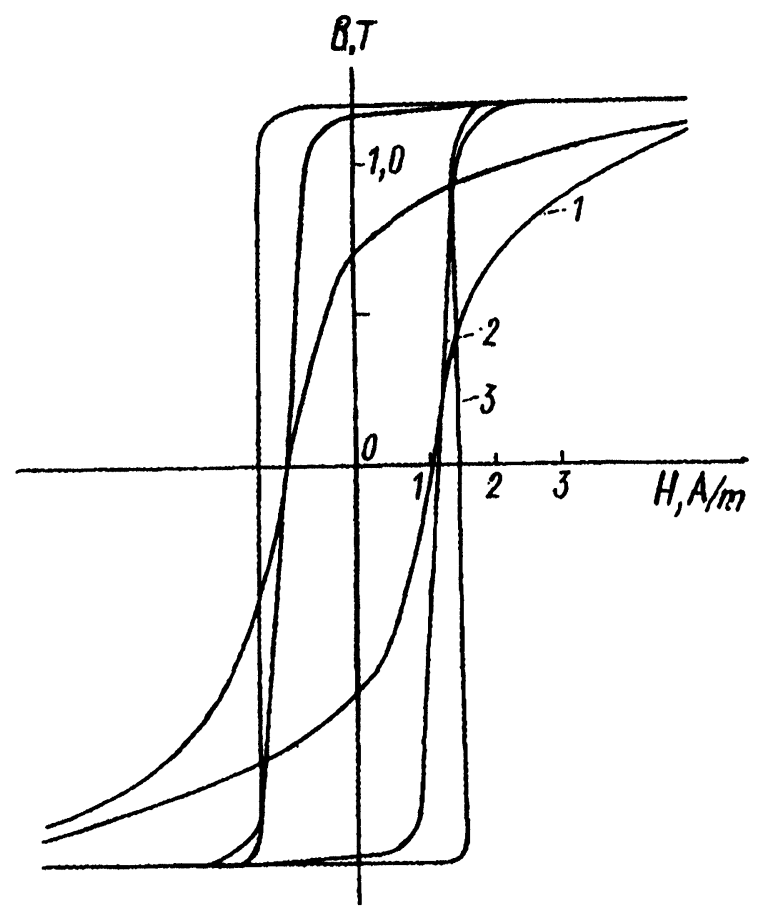

FIGURE 3 The hysteresis loops of samples in high frequency $(80 \mathrm{kHz})(1)$, alternating $(50 \mathrm{~Hz})(2)$, and direct (3) magnetic fields.

TABLE I The magnetic properties of FeCuNbSiB nanocrystalline alloy after TMT

\begin{tabular}{lccccc}
\hline Treatment & $\mu_{0} * 10^{-3}$ & $\mu_{\max } * 10^{-6}$ & $H_{\mathrm{c}}(\mathrm{A} / \mathrm{m})$ & $B r / B m$ & $P_{0.2 / 20000}(\mathrm{~W} / \mathrm{kg})$ \\
\hline $\mathrm{H}, \mathrm{f}=80 \mathrm{kHz}$ & 53 & 0.75 & 0.8 & 0.68 & 5.5 \\
$\mathrm{H}, \mathrm{f}=50 \mathrm{~Hz}$ & 37 & 1.20 & 0.8 & 0.96 & 8.0 \\
$\mathrm{H}, \mathrm{f}=0$ & 10 & 1.00 & 1.2 & 0.98 & 11.0 \\
\hline
\end{tabular}

the comparison of the hysteresis loops that TMT in a $80 \mathrm{kHz}$ magnetic field results in a higher remanent induction and lower coercivity, than after quenching in water. The magnetic losses $P_{0.2 / 20000}$ after TMT in a $80 \mathrm{kHz}$ magnetic field are four times lower than those after quenching in water. The results of the investigations confirm the conclusions by Herzer (1994), that in the nanocrystalline $\mathrm{FeCuNbSiB}$ alloy the induced anisotropy reaches a maximum equilibrium value if a 


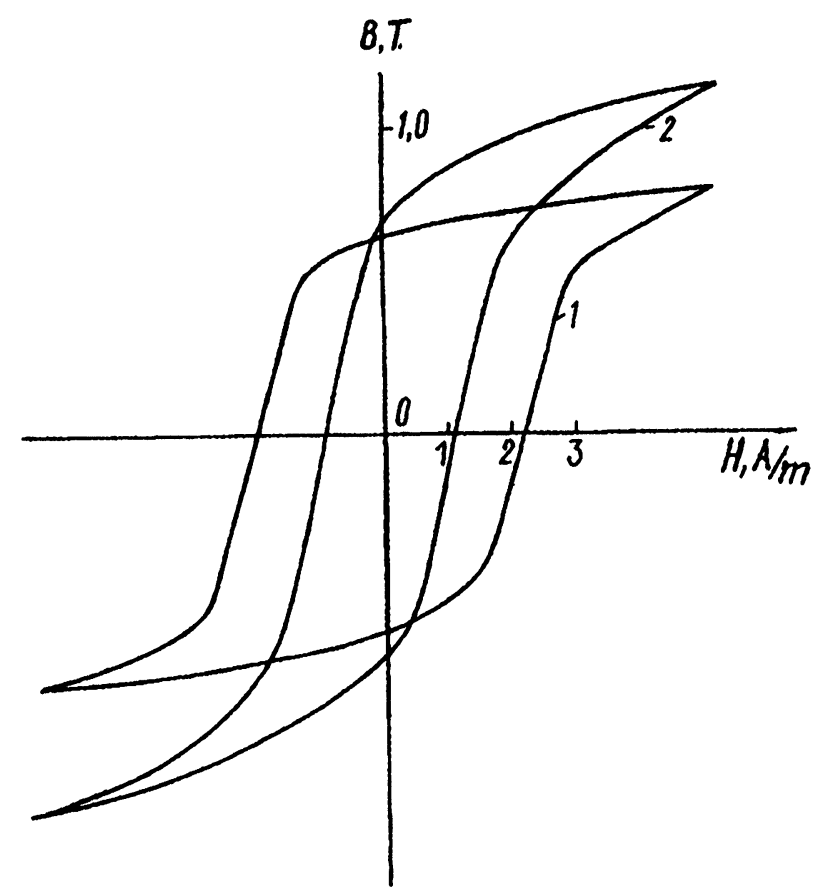

FIGURE 4 The hysteresis loops of samples after quenching in water (1) and high frequency TMT (2).

direct magnetic field was applied during the alloy transition from the amorphous to the nanocrystalline state. Apparently, the higher effectiveness of TMT in the nanocrystalline $\mathrm{FeCuNbSiB}$ alloy in comparison with the amorphous one is connected with a low value of a total magnetostriction of the nanocrystalline alloy. It is shown that the effect of TMT depends on the frequency of the magnetic field. The influence of TMT in a $80 \mathrm{kHz}$ magnetic field on the magnetic properties of nanocrystalline $\mathrm{FeCuNbSiB}$ alloy essentially differs from its influence in direct and alternating $50 \mathrm{~Hz}$ magnetic fields.

Apparently, this is due to the following reasons. In a high frequency $80 \mathrm{kHz}$ field magnetization reversal is realized by inhomogeneous rotation of magnetization. That is why the uniaxial anisotropy is not induced at TMT in such a field because of the lack of a preferred direction of magnetization, and a domain structure destabilization takes place. It leads to a nonrectangular hysteresis loop with the 
$\mathrm{Br} / \mathrm{Bm}$ ratio equal to 0.68 , and therefore to the increase of initial permeability and the decrease of the eddy-current component of the magnetic losses.

The uniaxial anisotropy is induced at TMT in a direct magnetic field and the hysteresis loop becomes rectangular (curve 3, Fig. 2) with a high value of $\mathrm{Br} / \mathrm{Bm}$. It reduces the initial permeability and makes the process of magnetization reversal "jumpy", which leads to sharp growth of the eddy-current component of magnetic losses. The magnetization reversal in a nanocrystalline alloy at TMT in an alternating $50 \mathrm{~Hz}$ field takes place by domain walls movement. TMT in the alternating field also leads to the development of uniaxial anisotropy, because the process of its inducing is an even effect, but its value is lower than after TMT in a direct field. Evidently, one can account for the abovementioned higher values of initial magnetic permeability and lower magnetic losses after TMT in an alternating $50 \mathrm{~Hz}$ field against TMT in a direct field. The traditional method of domain structure destabilization, namely, quenching in water from a temperature above the Curie point in the investigated alloy does not give optimal magnetic properties (curve 1, Fig. 4).

The sample after quenching has increased values of coercivity, magnetic losses and low values of the initial and maximum magnetic permeability. Evidently, this is due to the fact that the nanocrystalline $\mathrm{FeCuNbSiB}$ alloy contains $\alpha-\mathrm{Fe}-\mathrm{Si}$ grains, having magnetostriction $\lambda \sim 20 * 10^{-6}$.

As a result of quenching, large internal stresses appear in a sample, which leads to deterioration of both static and dynamic magnetic properties.

\section{CONCLUSIONS}

The influence of induced magnetic anisotropy on magnetic properties of the amorphous and nanocrystalline Fe73.5Cu1Nb3Si13.5B9 alloy was studied. It is shown that the effect of the TMT depends on the frequency of the magnetic field. It is established that domain structure destabilization of $\mathrm{FeCuNbSiB}$ nanocrystalline alloy at TMT in a high frequency magnetic field results in maximum decrease of magnetic losses and permeability increase, if a high frequency magnetic field is applied to a 
sample during the alloy transition from the amorphous to the nanocrystalline state.

\section{Acknowledgment}

The work was supported by the Russian Fund for Basic Research, pr. 96-02-16000.

\section{References}

Glazer, A.A., Shulika, V.V. and Potapov, A.P. (1992). Domain structure destabilization of amorphous alloys by thermomagnetic treatment in high frequency field. Doclady Academii Nauk (Russian), 324, 1191-1193.

Glazer, A.A., Shulika, V.V. and Potapov, A.P. (1994). Influence of induced magnetic anisotropy upon static and dynamic magnetic properties of amorphous soft magnetic alloys with different magnetostriction. The Physics of Metals and Metallography, 78, $45-51$.

Herzer, G. (1994). Magnetic field induced anisotropy in nanocrystalline $\mathrm{Fe}-\mathrm{Cu}-\mathrm{Nb}-\mathrm{Si}-\mathrm{B}$ alloy. Journal of Magnetism and Magnetic Materials, 133, 48-250. 